\title{
PRINCIPAL DISTRIBUTIONS FOR ALMOST UNPERTURBED SCHRÖDINGER PAIRS OF OPERATORS
}

\author{
DAOXING XIA
}

(Communicated by Paul S. Muhly)

\begin{abstract}
The relation between the principal distribution for an almost unperturbed Schrödinger pair operators $\{U, V\}$ and the unitary operator $W$ satisfying $V=W^{-1} U W$ is found.
\end{abstract}

\section{INTRODUCTION}

This paper is a continuation of the previous work [7]. Let $\mathscr{H}$ be a Hilbert space, $\{U, V\}$ be a pair of selfadjoint operators on $\mathscr{H}$ and $a \in \mathbb{R}$. This pair is said to be an almost unperturbed Schrödinger pair of operators [7] with parameter $a \neq 0$, if there is a trace class operator $D$ such that $i[U, V] \zeta=$ $a \zeta+D \zeta, \zeta \in M$, where $M \subset \mathscr{D}(U) \cap \mathscr{D}(V)$ is a linear manifold dense in $\mathscr{H}$ satisfying $U M \subset \mathscr{D}(V), V M \subset \mathscr{D}(U)$, and $M=(U-z I)^{-1} \mathscr{D}(V)$ or $M=(V-z I)^{-1} \mathscr{D}(U)$ for some $z \in \mathbb{C} \backslash \mathbb{R}$.

For this pair $\{U, V\}$, a cyclic one cocycle is given by the trace formula

$$
\begin{aligned}
& \operatorname{tr}\left(\left[e^{i s_{1} U} e^{i t_{1} V}, e^{i s_{2} U} e^{i t_{2} V}\right]-e^{i\left(s_{1}+s_{2}\right) U} e^{i\left(t_{1}+t_{2}\right) V}\left(e^{-i a s_{2} t_{1}}-e^{-i a s_{1} t_{2}}\right)\right) \\
& \quad=\tau\left(s_{1}+s_{2}, t_{1}+t_{2}\right)\left(e^{-i a s_{2} t_{1}}-e^{-i a s_{1} t_{2}}\right)
\end{aligned}
$$

where $[\cdot, \cdot]$ is the commutator, and the function $\tau$ may be written as

$$
\tau(s, t)=\operatorname{tr}\left(e^{i s U} \int_{0}^{t} e^{i \tau V} D e^{i(t-r) V} d \tau\right) / t a .
$$

The principal distribution for this pair $\{U, V\}$ is defined as

$$
G(x, y)=\frac{-a}{2 \pi} \iint e^{-i(s x+t y)} \tau(s, t) d s d t .
$$

Received by the editors January 2, 1990 and, in revised form, March 19, 1990; presented to the G.P.O.T.S., Albuquerque, New Mexico, April 19, 1990.

1980 Mathematics Subject Classification (1985 Revision). Primary 47A55; Secondary 47G05.

Key words and phrases. Principal distribution, Pincus principal function, Schrödinger pair of operators, perturbation, pseudo differential operator.

This work is supported in part by NSF grant DMS-8901506. 
The trace formula

$$
\begin{gathered}
\operatorname{tr}\left(i[\phi(U, V), \psi(U, V)]-a J_{a}(\phi, \psi)(U, V)\right) \\
\quad=-\frac{1}{2 \pi} \iint J_{a}(\phi, \psi) G(x, y) d x d y
\end{gathered}
$$

is proved in [7] for functions $\phi$ and $\psi$ in a certain class where $J_{a}(\phi, \psi)$ is a functional (see [7]) of $\phi, \psi$ and approaches the Jacobian of $\phi$ and $\psi$ as $a \rightarrow 0$. Without loss of generality, we assume that $a=1$.

In this paper, we study the principal distribution $G(x, y)$ for the almost unperturbed Schrödinger pair of operators $\{U, V\}$ under certain conditions. The main condition is that there is a unitary operator $W$ such that $V=W^{-1} U W$. When $V$ is in a certain class of integro-differential operators [7], these conditions are satisfied (see Lemma 2 and Theorem 5). We find that the principal distribution $G(x, y)$ may be expressed essentially by the integral kernel of the operator $W$ in a certain spectral representation (see Theorem 2 and Theorem $3)$.

In this paper, the Carey and Pincus theory $[2,3]$ of an almost commuting pair of unitary operators or selfadjoint operators plays an important role.

\section{PRincipal Distribution}

Let $\mathscr{L}^{1}$ and $\mathscr{L}^{2}$ be the trace class and Hilbert-Schmidt class of operators respectively.

Theorem 1. Let $\{U, V\}$ be an almost unperturbed Schrödinger pair of operators. If $\left(V-\mu_{0} I\right)^{-1}\left(U-\lambda_{0} I\right)^{-1} \in \mathscr{L}^{2}$ for some $\lambda_{0}, \mu_{0} \in \mathbb{C} \backslash \mathbb{R}$, then $\left[(V-\mu I)^{-1}\right.$, $\left.(U-\lambda I)^{-1}\right] \in \mathscr{L}^{1}$ for $\lambda, \mu \in \mathbb{C} \backslash \mathbb{R}$ and

$$
\operatorname{tr}\left[(V-\mu I)^{-1},(U-\lambda I)^{-1}\right]=0 \text { for } \lambda, \mu \in \mathbb{C} \backslash \mathbb{R} .
$$

Proof. (1) It is easy to see that $A=(V-\mu I)^{-1}\left(V-\mu_{0} I\right)$ extends a bounded operator and $B=\left(U-\lambda_{0} I\right)(U-\lambda I)^{-1}$ is a bounded operator.

Therefore $(V-\mu I)^{-1}(U-\lambda I)^{-1}=A\left(V-\mu_{0} I\right)^{-1}\left(U-\lambda_{0} I\right)^{-1} B \in \mathscr{L}^{2}$, for $\lambda, \mu \in \mathbb{C} \backslash \mathbb{R}$. Also $(U-\lambda I)^{-1}(V-\mu I)^{-1} \in \mathscr{L}^{2}$. Hence, the commutator

$$
\begin{aligned}
& {\left[(U-\lambda I)^{-1},(V-\mu I)^{-1}\right]} \\
& \quad=(U-\lambda I)^{-1}(V-\mu I)^{-1} C(V-\mu I)^{-1}(U-\lambda I)^{-1} \in \mathscr{L}^{1},
\end{aligned}
$$

where $C=-i(I+D) \in \mathscr{L}$ (cf. Lemma 1 of [7]).

(2) Form the Cayley transforms $u=(U+i I)(U-i I)^{-1}$ and $v=$ $(V+i I)(V-i I)^{-1}$. Then the commutator of unitary operators $[u, v]=$ $-4\left[(U-i I)^{-1},(V-i I)^{-1}\right] \in \mathscr{L}^{1}$. Let us employ the Carey and Pincus theory on the almost commuting pairs of unitary operators to the pair $\{u, v\}$.

With $\mathbb{Z}^{2}$ the 2-fold product of the set of integers, let $M\left(\mathbb{Z}^{2}\right)$ be the space of all complex Radon measures on $\mathbb{Z}^{2}$, for which

$$
\|m\|=\int_{\mathbb{Z}^{2}}(1+|s|)(1+|t|) d|m(s, t)|<+\infty .
$$


Let $\widehat{M}\left(\mathbb{Z}^{2}\right)$ be the collection of characteristic functions $F(\zeta, \eta), \zeta, \eta \in \mathbb{T}$, of the measures $m \in M\left(\mathbb{Z}^{2}\right)$ given by $F(\zeta, \eta)=\int_{\mathbb{Z}^{2}} \zeta^{l} \eta^{n} d m(l, n)$. From Theorem 5.10 and Remark 5.11 of [3], there is a Lebesgue integrable real function, called the principal function $g_{0}(\cdot, \cdot)$, such that

$$
\operatorname{tr}[f(u, v), h(u, v)]=\frac{i}{2 \pi} \iint g_{0}(\zeta, \eta) d f(\zeta, \eta) \wedge d h(\zeta, \eta)
$$

for $f, h \in \widehat{M}\left(\mathbb{Z}^{2}\right)$.

Let $\mathscr{M}\left(\mathbb{R}^{2}\right)$ be the collection of functions $f(x, y), x, y \in \mathbb{R}$, for which the functions $f(\phi(\zeta), \phi(\eta))$ as functions of $\zeta, \eta \in \mathbb{T}$ belong to $\widehat{M}\left(\mathbb{Z}^{2}\right)$, where $\phi(\zeta)=\operatorname{cotan} \arg \zeta / 2, \zeta \in \mathbb{T}$. Define

$$
g(x, y)=g_{0}\left((x+i)(x-i)^{-1},(y+i)(y-i)^{-1}\right), \quad x, y \in \mathbb{R} .
$$

Then by the Cayley transform, we have

$$
\operatorname{tr}[f(U, V), h(U, V)]=\frac{i}{2 \pi} \iint g(x, y) d f(x, y) \wedge d h(x, y)
$$

for $f, h \in \mathscr{M}\left(\mathbb{R}^{2}\right)$. This $g(\cdot, \cdot)$ may be regarded as the Pincus principal function associated to the almost unperturbed Schrödinger pair of operators.

(3) For $\lambda_{j}, \mu_{j} \in \mathbb{C} \backslash \mathbb{R}, j=1,2$, it is easy to see that the function $\left(x-\lambda_{j}\right)^{-1}\left(y-\mu_{j}\right)^{-1} \in \mathscr{M}\left(\mathbb{R}^{2}\right)$. By (6), we have

$$
\begin{aligned}
& \operatorname{tr}\left[\left(U-\lambda_{1} I\right)^{-1}\left(V-\mu_{1} I\right)^{-1},\left(U-\lambda_{2} I\right)^{-1}\left(V-\mu_{2} I\right)^{-1}\right] \\
& \quad=\frac{i}{2 \pi} \iint g(x, y) d\left(\left(x-\lambda_{1}\right)\left(y-\mu_{1}\right)\right)^{-1} \wedge d\left(\left(x-\lambda_{2}\right)\left(y-\mu_{2}\right)\right)^{-1}
\end{aligned}
$$

The left-hand side of (7) is zero, since $\left(U-\lambda_{j} I\right)^{-1}\left(V-\mu_{j} I\right)^{-1} \in \mathscr{L}^{2}$.

Define an analytic function $\phi(\lambda, \mu)$ of $\lambda, \mu \in \mathbb{C} \backslash \mathbb{R}$ by

$$
\begin{aligned}
\phi(\lambda, \mu) & =\frac{i}{2 \pi} \iint g(x, y) d \ln \frac{x-\lambda}{x-i} \wedge d \ln \frac{y-\mu}{y-i} \\
& =\frac{i}{2 \pi} \iint g(\zeta, \eta) \frac{d \zeta}{\zeta-\omega} \wedge \frac{d \eta}{\eta-z},
\end{aligned}
$$

where $\omega=(\lambda+i)(\lambda-i)^{-1}$ and $z=(\mu+i)(\mu-i)^{-1}$ (cf. (5.3) of [3]). By 
elementary calculus, the right-hand side of (7) equals

$$
\begin{aligned}
& \frac{1}{\left(\lambda_{1}-\lambda_{2}\right)\left(\mu_{1}-\mu_{2}\right)}\left\{\frac{\partial^{2} \phi}{\partial \lambda \partial \mu}\left(\lambda_{2}, \mu_{1}\right)-\frac{\partial^{2} \phi}{\partial \lambda \partial \mu}\left(\lambda_{1}, \mu_{2}\right)\right. \\
& \quad+\frac{1}{\left(\lambda_{1}-\lambda_{2}\right)}\left(\frac{\partial \phi}{\partial \mu}\left(\lambda_{1}, \mu_{2}\right)-\frac{\partial \phi}{\partial \mu}\left(\lambda_{2}, \mu_{2}\right)\right) \\
& \quad+\frac{1}{\left(\mu_{1}-\mu_{2}\right)}\left(\frac{\partial \phi}{\partial \lambda}\left(\lambda_{1}, \mu_{1}\right)-\frac{\partial \phi}{\partial \lambda}\left(\lambda_{1}, \mu_{2}\right)\right) \\
&-\frac{1}{\left(\lambda_{1}-\lambda_{2}\right)}\left(\frac{\partial \phi}{\partial \mu}\left(\lambda_{1}, \mu_{1}\right)-\frac{\partial \phi}{\partial \mu}\left(\lambda_{2}, \mu_{1}\right)\right) \\
&\left.-\frac{1}{\left(\mu_{1}-\mu_{2}\right)}\left(\frac{\partial \phi}{\partial \lambda}\left(\lambda_{2}, \mu_{1}\right)-\frac{\partial \phi}{\partial \lambda}\left(\lambda_{2}, \mu_{2}\right)\right)\right\} .
\end{aligned}
$$

Thus (8) equals zero. Putting $\lambda_{1}=\lambda, \lambda_{2}=\lambda+\varepsilon$ in (8) and letting $\varepsilon \rightarrow 0$, we get

$$
\frac{\partial^{3}}{\partial \lambda^{2} \partial \mu}\left(\phi\left(\lambda, \mu_{1}\right)+\phi\left(\lambda, \mu_{2}\right)\right)-\frac{2}{\left(\mu_{1}-\mu_{2}\right)} \frac{\partial^{2}}{\partial \lambda^{2}}\left(\phi\left(\lambda, \mu_{1}\right)-\phi\left(\lambda, \mu_{2}\right)\right)=0 .
$$

Putting $\mu_{1}=\mu, \mu_{2}=\mu+\varepsilon$ in (9) and letting $\varepsilon \rightarrow 0$, we have $\left(\partial^{5} /\left(\partial \lambda^{2} \partial \mu^{3}\right)\right) \times$ $\phi(\lambda, \mu)=0$. Similarly, we have $\left(\partial^{5} /\left(\partial \lambda^{3} \partial \mu^{2}\right)\right) \phi(\lambda, \mu)=0$. Therefore, the function $\left(\partial^{4} /\left(\partial \lambda^{2} \partial \mu^{2}\right)\right) \phi(\lambda, \mu)$ is a constant. Thus, there is a constant $k$ and functions $a(\lambda)$ and $b(\mu)$ such that $\left(\partial^{2} /(\partial \lambda \partial \mu)\right) \phi(\lambda, \mu)=k \lambda \mu+a(\lambda)+b(\mu)$. From (6), it is easy to see that $\left(\partial^{2} /(\partial \lambda \partial \mu)\right) \phi(\lambda, \mu)=\operatorname{tr}\left[(U-\lambda I)^{-1},(V-\mu I)^{-1}\right]$. Hence

$$
\operatorname{tr}\left[(U-\lambda I)^{-1},(V-\mu I)^{-1}\right]=k \lambda \mu+a(\lambda)+b(\mu) .
$$

(4) We have to prove that $k$ and $a(\lambda)+b(\mu)$ are zero. From (10), we have $\operatorname{tr}\left[(U-\lambda I)^{-2},(V-\mu I)^{-1}\right]=k \mu+a^{\prime}(\lambda)$. On the other hand, by (5), we have $\left|\operatorname{tr}\left[(U-\lambda I)^{-2},(V-\mu I)^{-1}\right]\right|$

$$
\begin{aligned}
& =2 \mid \operatorname{tr}\left(\left(U-\lambda_{0} I\right)^{2}(U-\lambda I)^{-3}\left(U-\lambda_{0} I\right)^{-1}(V-\mu I)^{-1} C(V-\mu I)^{-1}\left(U-\lambda_{0} I\right)^{-1} \mid\right. \\
& \leq 2\left\|\left(U-\lambda_{0} I\right)^{-1}(V-\mu I)^{-1} C(V-\mu I)^{-1}\left(V-\lambda_{0} I\right)^{-1}\right\|_{1}\left\|\left(U-\lambda_{0} I\right)^{2}(U-\lambda I)^{-3}\right\|,
\end{aligned}
$$

which approaches zero as $|\mathscr{I} \lambda| \rightarrow \infty$. Therefore $\lim _{\mathscr{f} \lambda \rightarrow \infty}\left(k \mu+a^{\prime}(\lambda)\right)=0$ which implies $k=0$.

It is easy to see that

$$
\begin{aligned}
\lim _{\mathscr{I} \lambda \rightarrow \infty} \operatorname{tr}\left((U-\lambda I)^{-1}(V-\mu I)^{-1} D(V-\mu I)^{-1}(U-\lambda I)^{-1}\right) \\
\quad=\lim _{\mathscr{I} \mu \rightarrow \infty} \operatorname{tr}\left((U-\lambda I)^{-1}(V-\mu I)^{-1} D(V-\mu I)^{-1}(U-\lambda I)^{-1}\right)=0
\end{aligned}
$$

We have to show that

$$
\lim _{\mathscr{f} \lambda \rightarrow \infty} \operatorname{tr}\left((U-\lambda I)^{-1}(V-\mu I)^{-2}(U-\lambda I)^{-1}\right)=0
$$


and

$$
\lim _{\mathscr{I} \mu \rightarrow \infty} \operatorname{tr}\left((U-\lambda I)^{-1}(V-\mu I)^{-2}(U-\lambda I)^{-1}\right)=0 .
$$

Let $\left\{e_{j}\right\}$ be an orthonormal basis for the Hilbert space $\mathscr{H}$. The series

$$
\sum_{j}\left(\left(V-\mu_{0} I\right)^{-1}(U-\lambda I)^{-1} e_{j},\left(V-\bar{\mu}_{0} I\right)(V-\bar{\mu} I)^{-2}(U-\bar{\lambda} I)^{-1} e_{j}\right)
$$

is dominated by the convergent series

$$
\begin{gathered}
\sum_{j} K\left\|\left(V-\mu_{0} I\right)^{-1}(U-\lambda I)^{-1} e_{j}\right\|\left\|(V-\bar{\mu} I)^{-1}(U-\bar{\lambda} I)^{-1} e_{j}\right\| \\
\leq K\left\|\left(V-\mu_{0} I\right)^{-1}(U-\lambda I)^{-1}\right\|_{2}\left\|(V-\mu I)^{-1}(U-\lambda I)^{-1}\right\|_{2}
\end{gathered}
$$

term by term, for $|\mathcal{F} \mu| \geq\left|\mathscr{F}_{\mu_{0}}\right|$, where $\|\cdot\|_{2}$ is the $\mathscr{L}^{2}$-norm,

$$
K=\max _{|\mathcal{F} \mu| \geq\left|\mathscr{F} \mu_{0}\right|}\left\|\left(V-\mu_{0} I\right)(V-\mu I)^{-1}\right\|
$$

and

$$
\lim _{\mathcal{I} \mu \rightarrow \infty}\left\|\left(V-\bar{\mu}_{0} I\right)^{-1}(V-\bar{\mu} I)^{-2}(U-\bar{\lambda} I)^{-1} e_{j}\right\|=0 .
$$

Therefore (13) holds. Similarly, we can prove (12). From (5), (11), (12), and (13), we have

$$
\lim _{\mathscr{I} \lambda \rightarrow \infty} \operatorname{tr}\left[(U-\lambda I)^{-1},(V-\mu I)^{-1}\right]=\lim _{\mathscr{I} \mu \rightarrow \infty} \operatorname{tr}\left[(U-\lambda I)^{-1},(V-\mu I)^{-1}\right]=0,
$$

which proves that $\lim _{\mathcal{I}_{\lambda \rightarrow \infty}}(a(\lambda)+b(\mu))=\lim _{\mathcal{I}_{\mu \rightarrow \infty}}(a(\lambda)+b(\mu))=0$. Thus $a(\lambda)+b(\mu)=0$. Theorem 1 is proved.

By the way, the Pincus principal function $g(x, y)$ must be of the form $\alpha(x)+$ $\beta(y)$.

For a Hilbert space $\mathscr{D}$, let $L^{2}(\mathbb{R}, \mathscr{D})$ be the Hilbert space of all measurable $\mathscr{D}$-valued funtion on $\mathbb{R}$ satisfying $(f, f)=\int\|f(x)\|_{\mathscr{D}}^{2} d x<\infty$.

Theorem 2. Let $\{U, V\}$ be an almost unperturbed Schrödinger pair of operators on $\mathscr{H}$. Suppose $U$ only has absolutely continuous spectrum with uniform multiplicity $n$, suppose there is a unitary operator $W$ such that

$$
V=W^{-1} U W
$$

and suppose $\left(V-\mu_{0} I\right)^{-1}\left(U-\lambda_{0} I\right)^{-1} \in \mathscr{L}^{2}$ for some $\lambda_{0}, \mu_{0} \in \mathbb{C} \backslash \mathbb{R}$. Then there is an auxiliary Hilbert space $\mathscr{D}$ with $\operatorname{dim} \mathscr{D}=n$, a measurable $\mathscr{L}(\mathscr{D})$-valued function $W(x, y)$ satisfying

$$
\iint \operatorname{tr}_{\mathscr{D}}\left(W(x, y)^{*} W(x, y)\right)\left(x^{2}+1\right)^{-1}\left(y^{2}+1\right)^{-1} d x d y<+\infty,
$$

a unitary operator $F$ from $\mathscr{H}$ onto $L^{2}(\mathbb{R}, \mathscr{D})$ such that

$$
\left(F U F^{-1} f\right)(x)=x f(x) \text {, }
$$


and

$$
\left(F W F^{-1} f\right)(y)=\int W(x, y) f(x) d x,
$$

for $f \in F \mathscr{D}(U)$ and distributions $a(x)$ and $b(y)$ such that the principal distribution

$$
G(x, y)=2 \pi \operatorname{tr}_{\mathscr{D}}\left(W(x, y)^{*} W(x, y)\right)+a(x)+b(y) .
$$

Proof. It is obvious that the auxiliary space $\mathscr{D}$ and the unitary operator $F$ exist such that $(16)$ is satisfied. We may assume that $\mathscr{H}=L^{2}(\mathbb{R}, \mathscr{D})$ and $F=I$.

There exists an $\mathscr{L}(\mathscr{D})$-valued measurable function $W_{0}(x, y)$ satisfying

$$
\iint \operatorname{tr}_{\mathscr{D}}\left(W_{0}(x, y)^{*} W_{0}(x, y)\right) d x d y<+\infty
$$

such that

$$
\left(\left(U-\mu_{0}\right)^{-1} W\left(U-\lambda_{0} I\right)^{-1} f\right)(y)=\int W_{0}(x, y) f(x) d x \text { for } f \in L^{2}(\mathbb{R}, \mathscr{D}),
$$

since $\left(U-\mu_{0} I\right)^{-1} W\left(U-\lambda_{0} I\right)^{-1}=W\left(V-\mu_{0} I\right)^{-1}\left(U-\lambda_{0} I\right)^{-1} \in \mathscr{L}^{2}$. Denote $W(x, y)=\left(x-\lambda_{0}\right) W_{0}(x, y)\left(y-\mu_{0}\right)$. Then (19) implies (15), and (20) implies (17) for $f \in F \mathscr{D}(U)$.

By Theorem 1 and (5), we have

$$
\begin{gathered}
\operatorname{tr}\left((U-\lambda I)^{-1}(V-\mu I)^{-1} D(V-\mu I)^{-1}(U-\lambda I)^{-1}\right) \\
=-\operatorname{tr}\left((U-\lambda I)^{-1}(V-\mu I)^{-2}(U-\lambda I)^{-1}\right) .
\end{gathered}
$$

The operator $(U-\lambda I)^{-1}(V-\mu I)^{-2}(U-\lambda I)^{-1}$ is a product of two $\mathscr{L}^{2}$ operators $(U-\lambda I)^{-1} W^{*}(U-\mu I)^{-1}$ and $(U-\mu I)^{-1} W(U-\lambda I)^{-1}$ with integral kernels $W(y, x)^{*}(x-\lambda)^{-1}(y-\mu)^{-1}$ and $W(x, y)(x-\lambda)^{-1}(y-\mu)^{-1}$ respectively. Therefore the right-hand side of $(21)$ is

$$
-\iint \operatorname{tr}_{\mathscr{D}}\left(W(x, y)^{*} W(x, y)\right)(x-\lambda)^{-2}(y-\mu)^{-2} d x d y .
$$

On the other hand, from (52) and (63) in [7], the left-hand side of (21) equals

$$
-\frac{1}{2 \pi} \iint G(x, y)(x-\lambda)^{-2}(y-\mu)^{-2} d x d y .
$$

Thus, the distribution $\psi(x, y)=G(x, y)-2 \pi \operatorname{tr}\left(W(x, y)^{*} W(x, y)\right)$ satisfies the condition

$$
\iint \psi(x, y)(x-\lambda)^{-2}(y-\mu)^{-2} d x d y=0
$$

for $\lambda, y \in \mathbb{C} \backslash \mathbb{R}$. From (22), we can prove that there are distributions $a(x)$ and $b(y)$ such that $\psi(x, y)=a(x)+b(y)$, which proves Theorem 2 . 
Remark 1 . The distribution $a(x)+b(y)$ can be determined uniquely by the property that the Fourier transform of $2 \pi \operatorname{tr}_{\mathscr{D}}\left(W^{*}(x, y)^{*} W(x, y)\right)+a(x)+b(y)$ is a bounded function (which is $\tau(s, t)$ ).

\section{A SIMPLER CASE}

In some special cases the distribution $a(x)+b(y)$ in Theorem 2 may be determined explicitly. A pair of selfadjoint operators $\{Q, P\}$ in $L^{2}(\mathbb{R}, \mathscr{D})$ is said to be Schrödinger pair of operators with multiplicity $\operatorname{dim} \mathscr{D}$, if $(Q f)(x)=$ $x f(x)$ for $f \in \mathscr{D}(Q)=\left\{f \in L^{2}(\mathbb{R}, \mathscr{D}):(\cdot) f(\cdot) \in L^{2}(\mathbb{R}, \mathscr{D})\right\}$ and $(P f)(x)=$ $i \frac{d}{d x} f(x)$ for $f \in \mathscr{D}(P)=\left\{f \in L^{2}(\mathbb{R}, \mathscr{D}) ; f\right.$ is absolutely continuous and $\left.f^{\prime} \in L^{2}(\mathbb{R}, \mathscr{D})\right\}$.

Lemma 1. Let $\{Q, P\}$ be the Schrödinger pair of operators on $L^{2}(\mathbb{R}, \mathscr{D})$ with $\operatorname{dim} \mathscr{D}<+\infty$. Then $(P-\mu I)^{-1}(Q-\lambda I)^{-1} \in \mathscr{L}^{2}$ for $\lambda, \mu \in \mathbb{C} \backslash \mathbb{R}$.

Proof. Without loss of generality, we may assume that $\mathscr{D}=\mathbb{C}$ and $\mathscr{I} \mu<0$. It is easy to see that $(P-\mu I)^{-1} f=-i \int_{-\infty}^{x} e^{i \mu(y-x)} f(y) d y$ and

$$
\left\|(P-\mu I)^{-1}(Q-\lambda I)^{-1}\right\|_{2}^{2}=\int \frac{d x}{|x-\lambda|^{2}} \int_{0}^{\infty} e^{2 \mathscr{F} \mu t} d t,
$$

for $\mathscr{I} \lambda \neq 0$. Therefore $(P-\mu I)^{-1}(Q-\lambda I)^{-1} \in \mathscr{L}^{2}$.

Theorem 3. Let $\{U, V\}$ be an almost unperturbed Schrödinger pair of operators on $\mathscr{H}$ satisfying the condition that there is a unitary operator $\Omega$ on $\mathscr{H}$ satisfying $\Omega-I \in \mathscr{L}^{1}$ such that $\left\{U, \Omega^{-1} V \Omega\right\}$ is unitarily equivalent to the Schrödinger pair of operators with multiplicity $n<+\infty$. Then the conclusion of Theorem 2 holds and, furthermore,

$$
G(x, y)=2 \pi \operatorname{tr}\left(W(x, y)^{*} W(x, y)\right)-n .
$$

Proof. Without loss of generality, we may assume that $\mathscr{H}=L^{2}(\mathbb{R}, \mathscr{D})$ with $\operatorname{dim} \mathscr{D}=n, U=Q$, and $\Omega^{-1} V \Omega=P$. It is easy to calculate that

$$
\left.\operatorname{tr}\left(e^{-i s t} e^{i s U} e^{i t V}-e^{i t V} e^{i s U}\right)=\operatorname{tr}\left(e^{i s Q}\left[e^{i t P}, \Omega\right] \Omega^{-1}\right)\right)\left(1-e^{-i s t}\right),
$$

and hence

$$
\tau(s, t)=\operatorname{tr}\left(e^{i s Q}\left[e^{i t P}, \Omega\right] \Omega^{-1}\right) .
$$

The unitary operator $\Omega$ may be written as

$$
\Omega=I+\sum_{j} \lambda_{j} P_{j},
$$

where $P_{j} f=\left(f, \eta_{j}\right) \eta_{j},\left\{\eta_{j}\right\}$ is an orthonormal set and $\left\{\lambda_{j}\right\}$ are the eigenvalues of $\Omega-I$, which must satisfy $\left|\lambda_{j}\right|^{2}+2 \mathscr{R} \lambda_{j}=0$ and $\sum\left|\lambda_{j}\right|<+\infty$, since $\Omega-I \in \mathscr{L}^{1}$. Therefore

$$
\begin{aligned}
\tau(s, t)= & -\sum \int e^{i s x}\left(\bar{\lambda}_{j}\left(\eta_{j}(x-t), \eta_{j}(x)\right)_{\mathscr{D}}+\lambda_{j}\left(\eta_{j}(x), \eta_{j}(x+t)\right)_{\mathscr{D}}\right) d x \\
& -\sum \lambda_{j} \bar{\lambda}_{k} \int e^{i s x}\left(\eta_{j}(x), \eta_{k}(x)\right)_{\mathscr{D}} d x \int\left(\eta_{k}(x-t), \eta_{j}(x)\right)_{\mathscr{D}} d x .
\end{aligned}
$$


From (3) and (26), it is easy to calculate that

$$
\begin{aligned}
G(x, y)= & 2 \sqrt{2 \pi} \sum \mathscr{R}\left(\lambda_{j}\left(\eta_{j}(x), \tilde{\eta}_{j}(y)\right) e^{i x y}\right) \\
& +2 \pi \sum \lambda_{j} \bar{\lambda}_{k}\left(\eta_{j}(x), \eta_{k}(x)\right)\left(\tilde{\eta}_{k}(y), \tilde{\eta}_{j}(y)\right),
\end{aligned}
$$

where $\tilde{\eta}_{j}(\cdot)$ is the Fourier transform of $\eta_{j}(\cdot)$.

For $b \in \mathscr{D}$, let $b^{*}$ be the functional defined by $b^{*} a=(a, b)$. It is easy to see that $V=\left(\Omega \mathscr{F}^{-1}\right) U(\Omega \mathscr{F})^{-1}$, where $\mathscr{F}$ is the Fourier transform (as an operator on $\left.L^{2}(\mathbb{R}, \mathscr{D})\right)$ defined by

$$
(\mathscr{F} f)(x)=s-\lim _{N \rightarrow \infty} \frac{1}{\sqrt{2 \pi}} \int_{-N}^{N} f(t) e^{i t x} d t .
$$

Therefore the operator $W$ in (14) may be chosen as $\mathscr{F} \Omega^{-1}$. Thus

$$
W(x, y)=\frac{1}{\sqrt{2 \pi}}\left(e^{i x y}+\sqrt{2 \pi} \sum \bar{\lambda}_{j} \tilde{\eta}_{j}(y) \eta_{j}(x)^{*}\right) .
$$

From (27) and (28), (23) follows.

\section{INTEGRO-DIFFERENTIAL MODEL}

In this section, we consider the integro-differential model (cf. $\S 4$ of [7]) of the almost unperturbed Schrödinger pair of operators.

Lemma 2. Let $\{U, V\}$ be an almost unperturbed Schrödinger pair of operators on $\mathscr{H}$ satisfying

$$
0 \leq D \in \mathscr{L}^{1} \quad \text { or } \quad 0 \geq D \in \mathscr{L}^{1}
$$

and

$$
\int_{-\infty}^{0} e^{i s U} D e^{-i s U} \in \mathscr{L}(\mathscr{H}) \quad \text { or } \quad \int_{0}^{\infty} e^{i s U} D e^{-i s U} d s \in \mathscr{L}(\mathscr{H}) .
$$

If $U$ has only absolutely continuous spectrum with finite multiplicity, then $(V-\mu I)^{-1}(U-\lambda I)^{-1} \in \mathscr{L}^{2}$ for $\lambda, \mu \in \mathbb{C} \backslash \mathbb{R}$.

Proof. By Theorem 2 of [7], we may assume that $\mathscr{H}=L^{2}(\mathbb{R}, \mathscr{D}), U=Q$, and $V=P+V_{1}$, where

$$
\left(V_{1} f\right)(x)= \pm \frac{\alpha(x)^{*}}{2 \pi i} \int \frac{\alpha(s) f(s) d s}{x-(s+i o)}
$$

for $f \in \mathscr{D}(Q)$, and $\alpha(\cdot)$ is an $\mathscr{L}(\mathscr{D} \rightarrow \overline{\operatorname{ran}(D)})$-valued measurable bounded function on $\mathbb{R}$. It is easy to see that $V_{1}$ is bounded, since

$$
\mathscr{P}: f \mapsto \frac{1}{2 \pi i} \int \frac{f(s) d s}{(\cdot)-(s+i o)}
$$

is an orthogonal projection. If $|\mathcal{F} \mu|>\left\|V_{1}\right\|$, then $\left\|V_{1}(P-\mu I)^{-1}\right\|<1$, since 
$\left\|(P-\mu I)^{-1}\right\|=|\mathcal{F} \mu|^{-1}$ and $(V-\mu I)^{-1}=(P-\mu I)^{-1} A(\mu)$, where $A(\mu)=$ $\sum_{n=0}^{\infty}\left(-V_{1}(P-\mu I)^{-1}\right)^{n}$ is a bounded operator. Thus $(U-\lambda I)^{-1}(V-\mu I)^{-1}=$ $(Q-\lambda I)^{-1}(Q-\mu I)^{-1} A(\mu) \in \mathscr{L}^{1}$ for $|\mathcal{F} \mu|>\left\|V_{1}\right\|$ and $\lambda \in \mathbb{C} \backslash \mathbb{R}$. By the technique of Step 1 in the proof of the Theorem 1 , we have $(V-\mu I)^{-1} \times$ $(U-\lambda I)^{-1} \in \mathscr{L}^{2}$ for all $\lambda, \mu \in \mathbb{C} \backslash \mathbb{R}$.

Theorem 4 (Jingbo Xia [8]). Let $\{U, V\}$ be an almost unperturbed Schrödinger pair of operators on $\mathscr{H}$ satisfying the conditions (29) and (30). If $U$ has only absolutely continuous spectrum with finite multiplicity. Then there is a finite number $c$ such that

$$
(V-\mu I)^{-1}-\left(V_{0}-\mu I\right)^{-1} \in \mathscr{L}^{1}
$$

for $|\mathscr{F} \mu|>c$, where $V_{0}$ is a selfadjoint operator satisfying the condition that $\left\{U, V_{0}\right\}$ is unitarily equivalent to the Schrödinger pair of operators.

Proof. As we stated in the proof of Lemma 2, we may assume that $\mathscr{H}=$ $L^{2}(\mathbb{R}, \mathscr{D}), U=Q, V=P+V_{1}$, and $V_{0}=P$. If $|\mathscr{J} \mu|>\left\|V_{1}\right\|$, then $I+(P-\mu I)^{-1} V_{1}$ is invertible and

$$
(V-\mu I)^{-1}-\left(V_{0}-\mu I\right)^{-1}=-\left(I+(P-\mu I)^{-1} V_{1}\right)(P-\mu I)^{-1} V_{1}(P-\mu I)^{-1} .
$$

We only have to show that

$$
(P-\mu I)^{-1} V_{1}(P-\mu I)^{-1} \in \mathscr{L}^{1},
$$

for $\mu \in \mathbb{C} \backslash \mathbb{R}$. Let $P_{+}$be the projection $\left(P_{+} f\right)(x)=1_{[0, \infty)}(x) f(x)$; then the operator $\mathscr{P}=\mathscr{F}^{-1} P_{+} \mathscr{F}$ where $\mathscr{F}$ is the Fourier transform. Let $M_{\alpha}$ be the operator $M_{\alpha} f=\alpha f$; then $V_{1}= \pm M_{\alpha}^{*} \mathscr{F}^{-1} P_{+} \mathscr{F} M_{\alpha}$. In order to prove (33), we only have to show that $\mathscr{F} M_{\alpha}(P-\mu I)^{-1} \mathscr{F}^{-1} \in \mathscr{L}^{2}$. Let

$$
\tilde{\alpha}(x)=s-\lim _{N \rightarrow \infty} \frac{1}{\sqrt{2 \pi}} \int_{-N}^{N} \alpha(t) e^{i t x} d t .
$$

Then this $\mathscr{L}(\mathscr{D} \rightarrow \overline{\operatorname{ran}(D)})$-valued measurable function $\tilde{\alpha}$ belongs to $L^{2}$, since the fact that $D \in \mathscr{L}^{1}$ implies that $\int \operatorname{tr}_{\mathscr{D}}\left(\alpha(t)^{*} \alpha(t)\right) d t<+\infty$. It is easy to see that the operator $\mathscr{F} M_{\alpha}(Q-\mu I)^{-1} \mathscr{F}^{-1}$ is defined by the integral kernel $(1 / \sqrt{2 \pi}) \tilde{\alpha}(y-x)(x-\mu)^{-1}$ which satisfies $\int\left\|\tilde{\alpha}(y-x)(x-\mu)^{-1}\right\|_{2}^{2} d x d y<+\infty$. Therefore $\mathscr{F} M_{\alpha}(P-\mu I)^{-1} \mathscr{F}^{-1} \in \mathscr{L}^{2}$, and the theorem is proved.

Theorem 5. Let $\{U, V\}$ be an almost unperturbed Schrödinger pair of operators on $\mathscr{H}$ satisfying conditions (29) and (30). If the operator $U$ has only absolutely continuous spectrum with finite multiplicity, then $\{U, V\}$ satisfies the conditions of Theorem 2.

Proof. By Birman, de Branges, and Kuroda Theorems (cf. $[1,4,5])$ and Theorem 4 , the wave operators $\Omega^{ \pm}\left(V, V_{0}\right)=s-\lim _{t \rightarrow \pm \infty} e^{i t V} e^{-i t V_{0}}$ exist and are complete, where $V_{0}$ is the operator in (32), since $P_{a c}(U)=I$ and consequently 
$P_{a c}\left(V_{0}\right)=I$. Let $\mathscr{F}$ be the Fourier transform satisfying $U=\mathscr{F} V_{0} \mathscr{F}^{-1}$ and write $W=\mathscr{F} \Omega^{+}\left(V, V_{0}\right)^{-1}$ or $\mathscr{F} \Omega^{-}\left(V, V_{0}\right)^{-1}$. Then $W$ is a unitary operator satisfying (14). This proves the theorem.

\section{ACKNOWLEDGMENT}

The author wishes to express his appreciation to Professor W. Arveson and Professor R. Douglas for their invitation to speak on [7] at the 1988 Summer Institute of the American Mathematical Society. The author is also grateful for the helpful discussion he had with Professor J. Pincus in New Hampshire, 1988. Without their encouragement this continuation of [7] would not have been possible.

\section{REFERENCES}

1. M. Birman, A criterion for existence of wave operators, Izv. Akad. Nauk. SSSR Ser. Mat. 27 (1963), 883-906.

2. R. W. Carey and J. D. Pincus, Mosaics, principal functions and mean motion in von Neumann algebras, Acta Math. 138 (1977), 153-218.

3. __ Almost commuting pairs of unitary operators and flat currents, Integral Equations Operator Theory 4 (1981), 45-122.

4. L. de Branges, Perturbation of self-adjoint transformation, Amer. J. Math. 84 (1962), 543580.

5. S. Kuroda, Perturbations of continuous spectra by unbounded operators. I, II, J. Math. Soc. Japan 11 (1959), 247-262; 12 (1960), 243-257.

6. M. Reed and B. Simon, Methods of modern mathematical physics III. Scattering theory, Academic Press, New York, 1979.

7. D. Xia, On the almost unperturbed Schrödinger pair of operators, Integral Equations Operator Theory 12 (1989), 242-279.

8. J. Xia, personal communication, 1989.

Department of Mathematics, Vanderbilt University, NashVille, Tennessee 37235 